\title{
El «problema» de la generación, la «generación» del problema. La producción social del problema de la empleabilidad juvenil en documentos de organismos públicos del mundo del trabajo
}

\author{
Alicia B. Gutiérrez \\ Instituto de Humanidades. Consejo Nacional de Investigaciones Científicas y Técnicas \\ (CONICET- UNC) \\ Universidad Nacional de Córdoba. Facultad de Filosofía y Humanidades \\ gutierre@ffyh.unc.edu.ar

\section{Gonzalo Assusa} \\ Instituto de Humanidades. Consejo Nacional de Investigaciones Científicas y Técnicas \\ (CONICET- UNC) \\ gon_assusa@hotmail.com
}

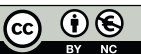

Recibido: 09-06-2014

Aceptado: 23-03-2015

\section{Resumen}

En este artículo, abordamos la construcción de la problemática del empleo juvenil en documentos de diagnóstico y formación de la Organización Internacional del Trabajo (OIT) y del Ministerio de Trabajo, Empleo y Seguridad Social de Argentina (MTESS), desde una perspectiva sociológica de la condición juvenil. Sobre la base de una investigación fundamentalmente cualitativa en un barrio de la ciudad de Córdoba (Argentina), nuestros análisis de las fuentes documentales giran en torno a los supuestos teórico-epistemológicos de estos discursos, así como también a la dinámica práctica de la noción de empleabilidad en la intervención política de esta problemática.

Palabras clave: sociología de la condición juvenil; políticas de empleo; estrategias laborales; clases populares; competencias laborales

Abstract. The "problem» of the generation, the "generation" of the problem: The social production of the problem of youth employability in public agencies documents of the working world

This article deals with the construction of the problem of youth employment in the diagnostic and training documents of the International Labour Organization (ILO) and the Labour, Employment and Social Security Ministry of Argentina (MTESS), based on a sociological perspective of the youth condition. This analysis is based on a fundamentally qualitative research in a neighborhood of the city of Córdoba (Argentina). Our study of the documentary sources refers to the theoretical and epistemological assumptions of these speeches, and also to the practical dynamic of the concept of employability in the political intervention in this problem.

Keywords: sociology of youth condition; employment policies; labor strategies; popular classes; job skills 


\section{Sumario}
1. Introducción
4. Reflexiones finales
2. La activación de la sociedad. Referencias bibliográficas
De la asistencia a la promoción Fuentes

3. La problemática del empleo juvenil para la OIT y el MTESS

\section{Introducción}

En este artículo, abordamos la construcción de la problemática del empleo juvenil en documentos de diagnóstico y formación de la Organización Internacional del Trabajo (OIT) y del Ministerio de Trabajo, Empleo y Seguridad Social de Argentina (MTESS) desde una perspectiva sociológica de la condición juvenil.

Este estudio forma parte de una investigación más amplia sobre la configuración de la cultura laboral en jóvenes de sectores populares de un barrio periférico de la ciudad de Córdoba (Argentina). En este marco, se ha realizado investigación sobre el terreno en distintos espacios institucionales vinculados a la formación para el empleo, incluida la principal política laboral para jóvenes en Argentina: el Programa Jóvenes con Más y Mejor Trabajo (PJMYMT) del MTESS. Así, algunos de los análisis sobre los materiales de formación y difusión de estos organismos estarán mediados por la descripción de las actualizaciones y los desplazamientos de sentido que operan los agentes técnicos que están a cargo del funcionamiento del programa en el territorio cordobés.

Nuestra intención es aportar elementos no solo para pensar el análisis de las prácticas y los dispositivos de la juventud, sino también para problematizar sobre la producción misma de la relación entre jóvenes y trabajo, en el particular contexto de la Argentina de la postconvertibilidad ${ }^{1}$. Luego de una década de aplicación de políticas neoliberales y de un régimen de acumulación con eje en la valorización financiera (Basualdo, 2006), el viraje operado en el año 2003 es objeto de múltiples discusiones. Dejando de lado la reconstrucción de las perspectivas encontradas, acerca del signo del crecimiento y de la profundidad de los cambios, existe cierto acuerdo respecto al proceso de paro forzoso: disminución del desempleo masivo, que llega a estacionarse alrededor de 2007 en torno a los 7 u 8 puntos y que varía levemente por los ciclos de la crisis internacional (Neffa et al., 2010). Esta mengua se hizo efectiva para todos los grupos de edad, aunque la brecha entre el desempleo de adultos (de 26 a 59 años) y el de jóvenes (de 15 a 25) aumentó a lo largo del período. En un con-

1. Luego de la crisis que Argentina atraviesa en el año 2001, y a través de un período de transición que llega hasta el 2003, la dinámica del mercado de trabajo y de la economía en general se estabiliza, lo cual da lugar a nuevas lógicas de acumulación y reproducción social. Algunos autores han llamado a este período la postconvertibilidad argentina (González Bombal et al., 2010). 
texto como este, signado por una serie de tendencias estructurales en las que aquí no profundizaremos, los modos de gestión gubernamentales del trabajo viraron desde un control - fundamentalmente paliativo- del desempleo hacia una gestión de la empleabilidad. Este salto, conceptualizado como el paradigma de políticas activas de empleo (Neffa, 2012), implica una manera distinta de producir (diagnosticar y generar propuestas para) la cuestión del empleo en condiciones estructurales diferentes a las de la década de 1990, además de implicar la habilitación de mecanismos de intervención también distintos. Desde nuestra perspectiva, y tal como intentaremos mostrar, en esta nueva red de dispositivos, la cuestión juvenil tiene una presencia fundamental como supuesto, como población objeto y como elemento de un marco discursivo hegemónico que regula las relaciones de lucha simbólica entre los distintos sectores sociales.

Comenzaremos analizando el paradigma de las políticas activas de empleo y los supuestos que plantean en torno a la juventud. Luego, explicitando la perspectiva analítica para el abordaje de la construcción social del problema del empleo juvenil, examinaremos los materiales antes descriptos en torno a tres núcleos de sentido:

a) La producción de la juventud como carencia, como peligro y como proyecto, en tanto modo de clasificación de estos organismos.

b) El supuesto de individualidad como modelo de persona que subyace en estos diagnósticos y pone en jaque la intervención frente a ciertas lógicas de la cultura popular juvenil.

c) La dinámica práctica de la noción de empleabilidad como eje conceptual de acción sobre la problemática del empleo juvenil.

Finalmente, hipotetizamos sobre algunas implicancias acerca del modo de generación (Martín Criado, 1998) de sujetos jóvenes puesto en juego en un dispositivo basado en el diagnóstico de la cultura del trabajo.

\section{La activación de la sociedad. De la asistencia a la promoción}

Ante las fuertes transformaciones estructurales en el mercado laboral de la postconvertibilidad, las políticas de empleo en Argentina pasaron de las intervenciones indemnizatorias a la movilización de la oferta de trabajo y la creación de empleo (Jacinto, 2010). Aunque distan de constituirse en un universo homogéneo, podemos señalar ciertas características fundamentales de las políticas activas de empleo, tomando en cuenta su principal manifestación, el PJMYMT.

Por un lado, la aplicación de este paradigma (que no se agota en el ámbito de la política pública) habría surgido en el contexto de emergencia de toda una nueva institucionalidad de mediación (Jacinto y Millenaar, 2009), propia de los contextos de heterogeneización, desafiliación, desestabilización e incertidumbre de los procesos de transición de los jóvenes hacia la (adulta) vida laboral. Tal 
y como lo entienden estos autores, los programas orientados por la activación surgen para apuntalar un momento de la trayectoria juvenil que, en un pasado ideal, se habría dado de manera ordenada e inclusiva para todos los jóvenes por igual.

Por otra parte, como muchas de las investigaciones señalan, el paradigma de las políticas activas está centrado en la regulación y formación de aspectos subjetivos, tales como la formación actitudinal, de mejoramiento de la autoestima, de incentivo a la motivación para el trabajo (Pérez, 2013) y de habilidades comunicacionales e interaccionales para el mundo laboral, a partir de intervenciones personalizadas y de procesos de acompañamiento permanente (Jacinto y Millenaar, 2009; Darmon et al., 2006).

Esta estrategia más amplia de subjetivación sienta sus bases en un diagnóstico orientado por la noción de empleabilidad. Proponiendo una articulación particular entre los elementos estructurales y subjetivos del mercado de trabajo, se entiende que el problema del empleo juvenil debe explicarse, por un lado, por el desarreglo de expectativas de los jóvenes inempleables y las demandas laborales reales (Salvia, 2008) y, por el otro, por un déficit de competencias o, en otras palabras, de capital humano y de educación (Salvia, 2013), según lo entienden las conceptualizaciones de la OIT, el Banco Interamericano del Desarrollo (BID) y el Banco Mundial.

En este sentido, la perspectiva de intervención habilitada por este diagnóstico es la de la formación (subjetiva, de valores, de actitudes). Sin embargo, mientras que, en la década de 1990, esta se focalizaba fundamentalmente en la capacitación, el cambio de siglo - y de condiciones- produjo un fuerte viraje hacia la perspectiva de orientación e inducción al mundo del trabajo (Pérez, 2013; Jacinto, 2010; Jacinto, 2008). En esta construcción del problema, la política activa ya no identificaría una falta de "competencias técnicas» específicas en los jóvenes vulnerables para ocupar empleos calificados, sino más bien un déficit de "competencias básicas y transversales», para la búsqueda, la consecución y el mantenimiento de empleos. Aquellas competencias que se adquieren en la vida familiar y escolar "normal», que son, justamente, más complejas de generar, pues son aprehendidas como «naturales». Esta intervención, mucho más adaptada a un mercado de trabajo flexibilizado, promueve - en consecuencia - la formación de competencias flexibles (ya sin la figura del saber específico en términos de oficio) para desempeñarse en un mundo laboral signado por la incertidumbre (Jacinto y Millenaar, 2009: 71).

A partir de esta reconstrucción, consideramos que la intervención mediada por el diagnóstico de la empleabilidad produce la problemática de empleo juvenil en relatos, discursos y prácticas en torno a jóvenes vulnerables, faltos de cultura del trabajo, verdadera instancia legitimante de la dignidad como persona, del estado de ciudadanía y de la percepción de beneficios económicos. Así, la intervención en términos de "formación laboral» se basa en una serie de supuestos acerca de la juventud de determinados sectores sociales, una generación "perdida", sin valores, in-competente e in-empleable. 


\section{La problemática del empleo juvenil para la OIT y el MTESS}

Tanto la OIT como el MTESS defienden la necesidad de aplicar políticas de empleo específicas para jóvenes y basan su defensa en esta lectura de la realidad. Los textos que aquí analizamos comprenden tanto resúmenes ejecutivos de reuniones y congresos como diagnósticos y documentos de trabajo, boletines, etc. La mayor parte está constituida por materiales de formación realizados específicamente para la capacitación de docentes, talleristas y agentes del equipo técnico del PJMYMT en lo concerniente a la noción de empleabilidad, intermediación laboral, proyecto ocupacional, orientación ocupacional, etc.

Aunque estos textos presentan matices, grados de especificidad y de particularidad diferenciales, además de tener procedencias diversas (algunos están producidos por la misma OIT, otros son encargados a fundaciones u originados en el Ministerio, unos se refieren a la realidad nacional y otros a la regional), nos centraremos aquí en sus líneas de continuidad, dado que nos interesa establecer un horizonte de posibilidad para las significaciones y la producción de sentido en torno al problema del empleo juvenil.

La orientación teórico-académica de estos documentos analizados reconoce permanentemente el carácter "heterogéneo» de la juventud y la mediación operada por los contextos "políticos, económicos, culturales y sociales», con lo cual se evita caer en la frecuente homogeneización de la situación. Ahora bien, ¿a quiénes van dirigidas las políticas de empleo para «jóvenes»? ¿De qué jóvenes se habla cuando se diagnostica la problemática de empleo juvenil?

\subsection{La producción social de los problemas públicos}

Partimos de la noción de que la relación de los jóvenes con el empleo como problema se aleja mucho de ser un dato obtenido de la realidad, y menos aún de presentar una causalidad evidente, aunque los diagnósticos y los dispositivos de estos organismos así lo necesiten sostener (Chaves, 2010). Realizando una crítica homóloga a la que Bourdieu practica contra la ilusión sustancialista en el análisis de las clases sociales (1990a), Martín Criado (1999) sostiene que la sociología de la juventud debe, antes que suponerla como grupo social efectivamente existente y actuante como agente colectivo, analizar las condiciones sociales de su producción: pensarla como una clase (de edad) en el papel. Mientras que la juventud se volvió visible para la agenda pública y de investigación como problema social (Chaves, 2010; Cajías, 1999), la sociología de la condición juvenil debe dar cuenta de la producción de este problema como parte del proceso de construcción de su objeto. Tal como sostiene Salvia, «[...] el problema no escapa a los procesos de construcción social de los temas de agenda» (2008: 13).

Siguiendo a Remi Lenoir, entender la «edad» como producto de luchas entre generaciones sirve también para pensar la definición del problema del empleo juvenil en el marco de las luchas de clasificación, de la división social del trabajo como trabajo social de división o, en otras palabras, del surgimiento del problema social como la construcción fenomenológica de la realidad 
(Lenoir, 1993). En este sentido, detenerse sobre este cuerpo de documentos implica analizar la génesis del problema de la empleabilidad juvenil, en términos de su reconocimiento y de su legitimación por las designaciones oficiales y estatales, así como también por la fuerza del discurso de los agentes intermediarios, de sabios y de expertos (Lenoir, 1993), considerando su interés estructural en la producción de problemas (Martín Criado, 2005: 90) y los efectos prácticos de las categorizaciones o clasificaciones tales como activo/inactivo, empleable/inempleable (Bourdieu, 1990b).

La producción del problema juvenil en relación con el mundo laboral, como lo plantea Martín Criado, implica tanto la producción de agentes con las disposiciones necesarias para ocupar determinadas posiciones en el campo (con formas determinadas de relacionarse con el empleo, de identificarse, de concebir posibilidades sociales, etc.), como la producción del campo y de las estrategias sociales factibles en él. El «mercado de trabajo», así comprendido, funciona como campo de estrategias materiales por el control de los recursos, pero también de estrategias simbólicas por la producción de valor de sujetos, objetos y prácticas (Martín Criado, 1998).

\subsection{Los supuestos sobre la juventud y el empleo}

\subsubsection{La carencia y la peligrosidad}

Recuperando el planteo de Chaves (2005), los jóvenes a los que hacen referencia estos documentos podrían ser caracterizados como «negados y negativizados». Los define un discurso de clausura que comienza describiendo a los jóvenes «vulnerables» — población objeto de las políticas activas de empleo-, por la negación de su capacidad de agencia y por la habilitación de «intervenciones paternalistas» (Chaves, 2005).

Así, estos jóvenes se construyen como faltos de experiencia, «desertores» escolares y con fuertes deficiencias en su educación. El abandono de la escuela combinado con una inserción «temprana» en el mercado de trabajo generarían una tendencia a la inestabilidad en el empleo, así como también una deficiente socialización en determinados hábitos y prácticas propios de la vida institucional: reconocimiento de la autoridad, concentración, atención, seguimiento de normas, etc.

Por otra parte, estos jóvenes vulnerables presentarían déficits en cuanto al modo de pensamiento racional y a las posibilidades de hacer elecciones «claras» para su trayectoria. Algunas veces, signados por la indecisión (los textos señalan el carácter negativo de la oscilación y el no sostenimiento de una planificación previamente definida a lo largo del tiempo) o por falta de «realismo», los jóvenes definirían proyectos inalcanzables o saltarían de un proyecto a otro sin solución de continuidad:

Planificación de proyectos que no se basan en el contexto ni en la situación actual del joven. Esta es una situación muy común, jóvenes que tienen más de 18 años que juegan al fútbol con amigos y quieren ser jugadores profesionales, llegar a un gran club; o jóvenes que aspiran a ser dueños o gerentes de importantes empresas, 
o que han dejado el secundario en segundo año y se proyectan como «abogadas», "contadores», etc. (MTESS - Manual orientación ocupacional)

Según los documentos, la «vulnerabilidad» de la juventud también se manifiesta en el plano subjetivo. Los jóvenes son caracterizados fundamentalmente por la «abulia», el «desinterés», la baja autoestima y la autoidentificación con las «necesidades» materiales:

La abulia y la falta de intereses pueden ser obstáculos para la proyección. Planificar un proyecto formativo laboral pondrá a los jóvenes en contacto con ideas y sentimientos íntimos de los que no siempre son conscientes. (MTESS - Manual orientación ocupacional)

Tal como podemos leer en los manuales, mientras que, en otros ámbitos (como el de la asistencia social), la necesidad económica puede ser un argumento válido, en el mundo laboral, y fundamentalmente en momentos tales como los de selección de personal, la exposición de necesidad (material o económica) y la manifestación de una disposición a trabajar de cualquier cosa puede resultar una estrategia inconveniente. Como luego veremos, estos diagnósticos proponen servicios específicos para producir disposiciones de planificación y competencias para la propia venta de los servicios laborales (para venderse, ofrecerse, en vez de pedir por trabajo ${ }^{2}$ ):

En los encuentros de trabajo con jóvenes vulnerables, es común escuchar frases tales como: «Yo solo quiero trabajar, trabajo de cualquier cosa», o "Lo que me ofrezca está bien, no me importa si me gusta». En estas frases, no se hace ninguna referencia a otras necesidades que no sean las económicas o las asociadas a la urgencia. Escuchamos que no se nombran ni los intereses, ni los proyectos de futuro (MTESS - Manual orientación ocupacional)

Por último, la familia aparece como un factor de vulnerabilización de estos jóvenes en varios sentidos. Por un lado, en términos de «cargas» o «responsabilidades» familiares ${ }^{3}$, que acotarían siempre la disponibilidad horaria (fundamentalmente en el caso de las mujeres) y generarían necesidades de reorganizar la vida doméstica para poder conseguir o sostener puestos laborales. Por otro lado, la familia aparece muchas veces como fuente de una serie de hábitos que los dispositivos propuestos intentan desterrar. En este caso, otro elemento recurrente en nuestro trabajo de campo es el relato de la juventud contemporánea como «segunda o tercera generación de desocupados en el país», en cuyos hogares, «los jóvenes nunca vieron a sus padres despertarse temprano

2. Distintas etnografías han explorado la capacidad de legitimación que ostenta la demostración de necesidad, en un momento particular de configuración de las políticas sociales y del campo de la asistencia (Zapata, 2005; Quirós, 2006, 2011).

3. Aquí, las negativizadas responsabilidades familiares se oponen a la promovida responsabilidad individual, valorada como competencia básica legítima para el mundo del trabajo. 
y salir a trabajar». El hogar, en general, aparece nominado en términos de problema: se dice que los beneficiarios provienen de familias "problemáticas» o de situaciones familiares «difíciles». Una de las dificultades que se generan en esta apreciación es la equivalencia establecida entre «empleos informales e inestables» y situación de "no trabajo».

Bajo la misma lógica, y desde una visión adulta y sociocéntrica, los diagnósticos construyen la disponibilidad de saberes acopiados por los jóvenes en sus propias experiencias y en las trayectorias familiares, fundamentalmente insertas en la economía informal, como un tipo de formación que deben desaprender para aumentar sus chances de inserción sociolaboral «formal».

Todas estas condiciones harían evidente la necesidad de generar instituciones, dispositivos e instancias de intervención, de mediación y de integración: «segundas oportunidades» para los jóvenes vulnerables. En caso contrario, ellos estarían mayormente expuestos a distintos tipos de peligrosidad. Como lo sostiene Chaves, podemos hablar de matrices discursivas de la "patología social» y del "pánico moral» (Chaves, 2005), en la medida en que pesa sobre los jóvenes vulnerables la presunción de, por un lado, desviaciones de distinta índole (formas de anomia, exclusión, «rebeldía» y desintegración) y, por el otro, delincuencia potencial:

Si bien no se trata de jóvenes desempleados en sentido estricto, el diagnóstico del desempleo como momento de una dinámica más amplia que articula recurrentemente períodos de empleo y desempleo vuelve necesaria su consideración, no solo por conformar un núcleo duro de exclusión de ámbitos fundamentales de integración, sino también por constituir el desenlace de un proceso marcado por el ingreso temprano al mercado laboral, el abandono de los estudios formales y los sucesivos fracasos en la búsqueda de una inserción laboral estable. Este constituye un grupo especialmente vulnerable a la anomia y el riesgo social, en muchos casos vinculado al desarrollo de formas ilegales y extralegales de subsistencia (MTESS - Diagnóstico desempleo juvenil)

Así, la juventud (especialmente si es pobre) se define como chivo expiatorio y depositario de una serie de miedos sociales, en gran parte asociados a sus «marcas subculturales» de clase, sus estéticas y consumos musicales diferenciales (Isla y Míguez, 2010).

Y no es necesario un razonamiento con argumentaciones ideológicas «de derechas» o "conservadoras». Antes bien, cabe perfectamente en una operatoria retórica de tipo "progresista»: de no otorgar la «educación» y la "cultura» necesarias, estos jóvenes no tendrían otra opción que la de caer en la delincuencia por culpa de una sociedad que los excluye. Sin embargo, como veremos en el apartado siguiente, el diagnóstico se apoya en supuestos vinculados a la voluntad individual o en su carencia, como elemento explicativo y de intervención fundamental de este cuerpo de textos.

\subsubsection{La proyección hacia el futuro}

Un segundo núcleo de significación fundamental asociado a la juventud vulnerable en este cuerpo de textos es el del joven como proyecto. Como exploraremos 
posteriormente, la noción de proyecto presenta una carga de sentido fundamental vinculada a la intervención que proponen estas instituciones, estableciendo cierta racionalización de las prácticas, es decir, promoviendo la definición de proyectos ocupacionales y la planificación de tareas para el logro de la inserción ocupacional plena. Por esto, la intervención se ve justificada, ya que la juventud aparece como el momento donde se cimentan las bases para la «autonomía» social y económica:

El proceso por el que está transitando el joven no se refiere solo a lo laboral ocupacional, sino que impacta sobre la percepción que tiene de sí mismo y la relación que sostiene con el medio que le rodea. El joven está construyendo los cimientos de su autonomía económica y social. Deberá, por lo tanto, elaborar este pasaje, que tiene su pasado en la infancia y su futuro en la adultez. El joven deberá desarrollar la capacidad para adecuarse a los cambios que esta vida adulta requiere. (MTESS - Manual acompañamiento)

En este sentido, y como una parte de las discusiones teóricas sobre la condición juvenil lo entiende (Chaves, 2010; Plesnicar, 2013), estos textos reconstruyen la juventud como «momento» de crisis vital, de «transiciones» hacia la vida adulta, de "encrucijadas» hacia la definición de una «identidad ocupacional». Como ha sostenido ya Chaves (2005), esta operación construye al sujeto joven como entidad incompleta, proyectada hacia el futuro y negada en su presente:

La juventud, por otro lado, marca un momento diferente. Los jóvenes saben que, al salir de la adolescencia, se encuentran ante la demanda de definir su futuro, entre otras cosas, a partir de la elección de una determinada actividad. Este momento es turbulento, pero igualmente constituye una instancia clave en la vida y en el proyecto vital de las personas. Es el momento en que el sujeto empieza a expresar con mayor claridad hacia donde quiere dirigir su vida, a enunciar con mayor evidencia cuáles son sus deseos y las opciones culturales, deportivas, sexuales que elige. El rompecabezas, entonces, comienza a armarse. Algunos elegirán una carrera para formarse en algún oficio o en alguna profesión. Otros aspirarán a posicionarse laboralmente y a no quedar afuera de las ofertas del mercado de trabajo. (MTESS - Manual acompañamiento)

A partir de ello, y considerando la conjunción de las significaciones de carencia, peligrosidad y proyecto, el tipo particular de intervenciones que se propone desde estos espacios sostiene la perspectiva de la formación y la orientación. Y todo esto se hace visible en las funciones del tutor ${ }^{4}$ en el PJMYMT.

La figura del tutor es definida como de «autoridad» potencial, aunque de carácter «flexible». Su misión de «acompañamiento» de la realización de los

4. El equipo técnico del PJMYMT, que es el encargado del sostenimiento del programa en el «territorio», está compuesto por cuatro funciones distintas: el orientador, una suerte de coordinador de todos los servicios del programa; el operador, responsable de la carga de datos y actualizaciones en el sistema informático de la Gerencia de Empleo; el relacionador, que se dedica a los proyectos de pasantías y vinculaciones con empresas, y el tutor, quien realiza el seguimiento de los jóvenes beneficiarios y quien tiene contacto directo con cada uno de ellos. 
proyectos ocupacionales tiene un sentido pedagógico fuertemente moral y conductual: la óptima realización de su tarea es la de actuar "con firmeza" pero «sin rigidez»:

Dentro de esta variedad de comportamientos, es deseable que el tutor sostenga con firmeza pero sin rigidez aquellos que son afines al papel que le toca desempeñar en el rol de entrevistador en el proceso de seguimiento, acompañamiento y apuntalamiento del proceso de los jóvenes. (MTESS - Manual acompañamiento)

Una de sus principales funciones es la de definir un «encuadre de trabajo»: un marco normativo, explicitado para el joven, de los términos en los que se desenvolverá su relación, pero que, además, será homólogo y servirá de referencia para la incorporación de un conjunto de reglas necesarias para interactuar y desenvolverse en el ámbito laboral (parte del déficit del joven vulnerable que arriba describimos).

Por último, la relación a construir entre el tutor y el joven beneficiario es, según lo establecen los manuales, un vínculo que aproveche la trama relacional familiar y social previa del joven, personalizando la interacción para acompañar un proceso fundamentalmente individual y subjetivo.

[...] en consecuencia, la realización de las actividades del módulo será, mayoritariamente, de forma individual. En este contexto, el asesoramiento personalizado por parte del capacitador o de la capacitadora constituirá un elemento de suma importancia (MTESS - Manual clubes de empleo)

La ambivalente autodefinición que los integrantes del equipo técnico de PJMYMT ensayan en las entrevistas, en términos de "técnico" y de "atención personalizada» responde, según entendemos, a una lógica de legitimación de sí mismos en los puestos de trabajo ocupados: si la primera - en términos de competencia certificada, saber legitimado y selección despersonalizada- les sirve para diferenciarse del conjunto de empleados públicos municipales (con quienes compartían dependencia institucional y espacial), sobre quienes pesa una fuerte descalificación en términos de "vagancia» $\mathrm{y}$ "acomodo", la definición de la propia tarea en términos de un «acompañamiento personalizado» les permite distinguirse de la práctica "punteril» de asistencia y entrega de dinero y bolsones de la década de 1990, en la cual los beneficiarios de la política social no serían sino un «número más».

Nuestro argumento se centra en mostrar que este tipo de intervenciones personalizadas y, en cierta medida, paternalistas (en el sentido político y filial de la palabra), de acompañamiento y apuntalamiento de trayectorias y transiciones, está basado en una construcción del sujeto joven como carente y «en transición». Como podremos observar, esta construcción, tanto del problema como de la intervención, se complementa con los supuestos del carácter «subjetivo» de la carencia y con las definiciones que impone el enfoque de la empleabilidad en este punto. 


\subsection{El supuesto de la subjetividad individual y el proyecto de la autonomía individual}

El POI (o Proceso de Orientación e Inducción al Mundo del Trabajo) es el servicio introductorio de los beneficiarios del PJMYMT. En el marco de este curso de dos meses de duración, al que los beneficiarios asisten tres veces por semana, el módulo más relevante para los fines del programa es el denominado Proyecto Formativo Ocupacional. En este espacio, los talleristas - docentes a cargo del módulo- acompañan el proceso de definición de un "proyecto» que orientará a los jóvenes y a sus tutores para el trayecto que cada beneficiario ensayará en el interior del programa. Según entendemos, esta instancia es una de las que condensa más claramente los lineamientos político-epistemológicos más relevantes del programa: el supuesto de la subjetividad individual como soporte de las prácticas que las políticas activas intentan intervenir, regular y orientar.

Así, la subjetividad individual de los jóvenes aparece como lugar del déficit de empleabilidad y, a la vez, como soporte de las intervenciones y la formación de hábitos que las políticas activas proponen (Darmon et al., 2006). Aquí señalaremos dos líneas fundamentales de esta intervención.

La primera está referida a las prácticas de autoconocimiento e introspección, que tienen un lugar central en los cursos y en los procesos de los que hablamos y que apuntan a la identificación de "fortalezas personales», de "expectativas y motivaciones» y al mejoramiento de la "autoestima», como algunas de las principales herramientas de los jóvenes para su inserción laboral:

[...] el taller prevé un conjunto de actividades orientadas a trabajar algunos aspectos de autoestima y a promover la reflexión para descubrir fortalezas personales, que pueden constituir atributos valorados en el mercado de trabajo (MTESS - Manual clubes de empleo)

A título individual, el desempleo prolongado puede significar una vida de subsistencia, la pérdida de autonomía personal y sentimientos de frustración, ira y/o baja autoestima. (OIT - Competencias)

Entendemos como autoconocimiento la capacidad de observación y reflexión acerca de uno mismo, focalizada en identificar motivaciones, aptitudes, gustos, comportamientos reiterados, reacciones y modalidad de resolución de problemas. (MTESS - Manual empleabilidad)

Son más difíciles de desarrollar aquellas que tienen que ver con actitudes: iniciativa, confianza en uno mismo, tenacidad, motivación. Para desarrollar los niveles de competencias más ligados a la personalidad, la vía privilegiada es el autoconocimiento. (MTESS - Manual empleabilidad)

La segunda remite a prácticas de racionalización de las habilidades y de las técnicas de interacción: en relación con lo antes descripto acerca de la construcción de la juventud-vulnerable-como-carencia, los documentos señalan con 
vehemencia la necesidad de introducir habilidades de administración y control de las emociones (tales como la frustración por no lograr inmediatamente los objetivos laborales abordados):

Las competencias relacionales suponen habilidades de comunicación, adaptabilidad, resolución de conflictos, control emocional, etc. La escena interpersonal pone en juego la actitud y la aptitud social de cada uno de los actores. 7-Administración de las emociones: manejo de emociones en diferentes situaciones. Nos referimos aquí al conjunto de emociones propias de la vida psíquica de todas las personas: alegría, tristeza, euforia, apatía, enojo, ira, compasión, angustia, ansiedad, etc. (MTESS - Manual empleabilidad)

Para esta construcción, la subjetividad ${ }^{5}$ funciona como soporte de una identidad ocupacional y, fundamentalmente, de un proyecto de vida no desarrollado, o subdesarrollado, y asociado a la autonomía y a la activación, como características positivas a trabajar en la población diagnosticada. Los documentos trabajados de la OIT y del MTESS pretenden introducir, a través de la serie de dispositivos que despliegan, una sistematicidad en las búsquedas laborales de los jóvenes vulnerables y una provisión de herramientas para desenvolverse en el mundo laboral formal. En este sentido, una de sus imágenes más recurrentes es la del trabajador como un individuo autónomo. Desde esta perspectiva, se entiende que las condiciones sociales de exclusión generan marcas subjetivas como la «baja autoestima» y la «pasividad», que funcionan como refuerzos de dicho proceso de exclusión.

Desde algunas perspectivas, se plantea que el desarrollo de la empleabilidad es responsabilidad primaria de cada persona, como decisor de su carrera laboral y director de su proyecto personal. Pero, en el caso de los jóvenes, si no cuentan con el acceso facilitado a la educación y a la capacitación laboral, tienen menos oportunidades de insertarse en el mercado formal de trabajo y esto refuerza la exclusión (MTESS - Manual empleabilidad)

Así, los materiales hacen fuerte hincapié en «mostrar» a estos jóvenes la posibilidad de «elegir sobre su propio futuro». Es importante remarcar el hecho de que «su destino no esté predefinido" y establecer que se requiere, por un lado, comprender la necesidad de renunciar a algunas aspiraciones y deseos

5. Esto no implica una omisión total de los factores estructurales o de las condiciones en el diagnóstico de la problemática de la empleabilidad. Sin embargo, la relación entre la dimensión subjetiva y objetiva no queda del todo explicitada en estos materiales y las actividades apuntan sobre todo a tener en consideración los límites que imponen las condiciones (familiares, sociales, culturales, económicas, del mercado de trabajo, etc.), pero siempre para intervenir sobre ellas y sobre las prácticas subjetivas. El enfoque que orienta esta estrategia presupone que los sujetos estamos condicionados por situaciones estructurales de contexto y que, a la vez, hay espacios de autonomía relativa - individual y colectiva - donde podemos «jugar» con la realidad. Así, el proyecto ocupacional es el resultado de una negociación entre los condicionamientos del entorno y el potencial de los sujetos, en términos de atributos e intereses (MTESS - Manual proyecto ocupacional). 
(como adulto, ya no puede hacerse «lo que se quiere») y, por el otro, planificar las búsquedas laborales. En otras palabras, introducir criterios de racionalización en este ámbito de la vida:

No obstante esto, incluirse en una actividad formativa y/o ocupacional será, para este joven, motivo de renuncias. Ya no podrá hacer todo lo que quiera, deberá aprender a respetar las reglas del trabajo, deberá experimentar progresivamente la relación entre esfuerzo, oportunidad, competencias y obstáculos personales. (MTESS - Manual acompañamiento)

La centralidad de la figura del "proyecto ocupacional» en el programa adquiere sentido, entonces, no solo como herramienta de trabajo de tutores y beneficiarios, sino también como experiencia de disciplinamiento en el uso del tiempo a medio y largo plazo. En parte, llegar a ser un individuo autónomo es poder renunciar a deseos y aspiraciones en el presente, en pos de un futuro de objetivos planificados, de inserción laboral:

Cuando la orientación se basa en la elección vocacional, el tiempo en juego es el futuro, el futuro entendido en el largo plazo. Se trabaja sobre una elección actual con consecuencia en el futuro y suele ser utilizada para que el joven adolescente elija una carrera, una profesión y todo el estilo de vida que esta conlleva. La vida cotidiana de una actriz, un contador, una enfermera y un técnico agrícola serán claramente distintas. El joven adolescente elige hoy la persona que será en el futuro. (MTESS - Manual acompañamiento)

Pero, por otra parte, la noción de autonomía se construye en relación con tres elementos fundamentales: el oficio o el saber laboral específico, la familia y el tutor. Mientras que la capacitación técnica «ata» — de alguna manera— al trabajador a las especificidades de un puesto laboral, la orientación y la formación para el mundo del empleo para los jóvenes vulnerables parte del supuesto de un mercado de trabajo flexibilizado, de cuyos puestos es también necesario ser autónomo. Ser autónomo siendo flexible:

Esta estrategia se apoya en la capacidad humana de formularse proyectos. Busca potenciar en las personas competencias (saberes, habilidades, actitudes) para mejorar su posición respecto del mundo laboral, especialmente en contextos de crisis, cambio e incertidumbre, que requieren de un mayor protagonismo, autonomía y flexibilidad. (MTESS - Manual proyecto ocupacional)

La familia, que aparecía ya como condicionante de los hábitos formados en referencia al joven vulnerable, surge nuevamente como una «carga» o «responsabilidad» que limita al joven para la consecución de un puesto de trabajo:

[...] por ejemplo, en muchas entrevistas, se indaga acerca del estado civil, el número de hijos, la estructura familiar, los gustos y pasatiempos, etcétera, datos que posibilitarán al entrevistador o a la entrevistadora evaluar las condiciones de disponibilidad del postulante o la postulante para cubrir el 
puesto de trabajo en cuestión. Por lo tanto, las respuestas a estas preguntas deben realizarse de manera tal que permitan destacar los recursos disponibles en cuanto a cada uno de estos aspectos, demostrando que ninguno de ellos podría transformarse en un impedimento para cumplir con las tareas y las responsabilidades propias del empleo al que se aspira, para evitar o neutralizar sesgos de discriminación hacia las personas con responsabilidades familiares y/o domésticas, en las respuestas a las preguntas arriba señaladas. También es recomendable intentar centrarse, primordialmente, en los aspectos vinculados con las competencias laborales y no en aquellos que pertenecen a la organización de la vida privada. (MTESS - Manual clubes de empleo)

Por último, los materiales promueven, entre el tutor y el beneficiario joven, la construcción de una relación que, aunque debe basarse en un vínculo personalizado que posibilite prácticas de acompañamiento y formación, debe cuidarse de no producir «dependencia» — actitud que, desde el paradigma moderno de la ilustración, corresponde a la «minoría de edad» de la humanidad (Foucault, 1985):

El tutor puede apuntalar al joven, sostenerlo en su proceso, pero debe ser él quien tome decisiones, quien realice las tareas correspondientes a su capacitación y a su inserción laboral. El tutor debe estar alerta en relación con los roles que los jóvenes le adjudican y los roles que, como contrapartida, sin querer, él puede llegar a asumir. En determinadas situaciones, él puede asumir roles que no beneficien el proceso que está realizando un determinado joven. (MTESS - Manual acompañamiento)

La relación de dependencia, aquí negativizada ${ }^{6}$ (Darmon et al., 2006: 44), aparece en los discursos de los agentes del equipo técnico y de los funcionarios del Ministerio como propios del estilo político «asistencialista» $\mathrm{y}$ "punteril» de la década de 1990 que hemos mencionado.

Lo mismo sucede con la familia, especialmente en el caso de las mujeres: el logro de la autonomía individual es, en gran parte, un proceso de independencia, de negociación y de escisión de mundos: el de lo laboral, por un lado, y el de lo familiar y doméstico, por otro. Sin embargo, nuestro trabajo de campo ha mostrado que la familia ha sido un espacio de referencia fundamental y uno de los principales recursos movilizados para la búsqueda laboral, aunque por vías informales. Para los empleos a los que muchos de los jóvenes que entrevistamos tuvieron acceso (servicio doméstico y cuidado de personas, en el caso de las mujeres, tareas ligadas a la construcción, en el caso de los varones), la formación de lazos de confianza fue fundamental ${ }^{7}$, respaldada por relaciones filiales o de vecindad. En el caso de las jóvenes, la materialidad misma de su

6. Martín Criado habla del ama de casa como un referente negativo entre los jóvenes, al límite de ser considerado un antisujeto, un «mantenido» (Martín Criado, 1998).

7. Vargas (2005) trabaja la importancia de la formación de lazos de "confianza», mediados por relaciones familiares y de etnicidad, en el contexto del trabajo en la obra (en el sector de la construcción). Recursos sociales y «confianza» son también fundamentales en los contextos de trabajo analizados por Lomnitz (1978 y 1979). 
proceso de empleo transcurre, muchas veces, en el hogar e involucra saberes desarrollados en el espacio doméstico.

Tal como venimos planteando, el tan denostado valor de la «dependencia» en el discurso de la OIT y el MTESS, asociado a la cultura asistencial y a la infantilidad, se positiviza en el marco de la cultura popular: «[...] se plantea la idea de que alguien es tal porque depende de otros, y en ese círculo de dependencias contrae obligaciones y derechos» (Míguez y Semán, 2006: 27), obligaciones movilizadas y habilitadas para la inserción laboral y social. Esta lógica colisiona, en alguna medida, con las nociones promovidas por estos materiales de formación, en términos de competencias y saberes certificados, de derechos universales, de selecciones legitimadas por el mérito individual ${ }^{8}$.

Por otra parte, encontramos que los jóvenes también valoran la autonomía en el proceso de trabajo propiamente dicho. Muchos de ellos responden, al ser consultados por las características de un «buen empleo», con la misma frase: "Un trabajo donde me dejen trabajar tranquilo», es decir, donde no tengan que soportar la presencia acuciante y el control permanente de un superior, como el «encargado». Sin embargo, al narrar sus experiencias, incluso aquellas que se encuentran en el marco de pasantías del programa, entienden que la actitud más útil en el mundo laboral no es la de la autonomía más exacerbada, la «iniciativa» y la "capacidad de negociación», sino la de la «sumisión activa»?: siempre que se termina una tarea, pedir una nueva, ofrecerse para ayudar o para limpiar. Pero, a la vez, y no menos importante, ante cualquier conflicto, «agachar la cabeza», demostrar que «uno no genera problemas», que no protesta, que siempre «cumple».

¿Cuán flexibles son los puestos a los que estos jóvenes efectivamente acceden? ¿Hablamos de flexibilidad del mercado de la misma forma que hablamos de flexibilidad de las tareas y los procesos de trabajo? ¿Cuánta autonomía les es concedida y cuánta necesitan los jóvenes vulnerables en el mundo laboral en el que se insertan?

\subsection{Teoría y práctica. La perspectiva de la empleabilidad}

Estos diagnósticos se orientan desde la noción de empleabilidad, conceptualmente emparentada con la de capital humano (Salvia, 2013; Martín Criado, 1999). Uno de los problemas que identificamos, y que atraviesa toda la argu-

8. Para una discusión conceptual acerca de los modelos de persona en las clases populares y en la cultura moderna, ver Dias Duarte (1995). Las «personas» en el mundo popular, a diferencia del individuo en la ideología moderna (Dias Duarte, 2004: 2; 1995: 24; 1994 : 83), estarían sujetas a lógicas culturales diferenciales y específicas, con una configuración moral alternativa (Semán, 2007) y un soporte fundamentalmente «relacional» y «holista» (Semán, 2006; Merklen, 2005).

9. Martín Criado (1998) analiza la estrategia de un grupo de jóvenes desprovistos de capital cultural legítimo que están de acuerdo en «humillarse» aceptando puestos de trabajo descualificados y manteniendo modestas sus exigencias, con la expectativa del «contradón» de la promoción en la empresa. De esta manera, se instalan ciertas expectativas de reciprocidad en las relaciones laborales, aun cuando la lógica «estrictamente» económica indique lo contrario. 
mentación de estos documentos, es que la idea de capital aparece aquí como un haber sustancializado y disponible, que no contempla su desigual distribución entre las distintas posiciones del espacio social (Gutiérrez, 2011). En este sentido, la promoción de las competencias de los jóvenes de baja empleabilidad como principal línea de intervención aparece como una inversión en la productividad de las empresas y, por lo tanto, en el crecimiento económico y el desarrollo social:

La OIT respalda el desarrollo de competencias con miras a mejorar la empleabilidad de los trabajadores, la productividad de las empresas y la capacidad integradora del crecimiento económico. (OIT - Competencias)

La empleabilidad contempla, entonces, una serie de saberes y competencias, aunque aquí nos centraremos específicamente en aquellos de índole «interaccional»:

Empleabilidad es el conjunto de competencias (conocimientos, habilidades, aptitudes y actitudes) que le permiten a una persona mantenerse en el mercado laboral o acceder a él. (MTESS - Manual empleabilidad)

Las competencias como centro de intervención permiten distinguir la perspectiva de la "orientación", de aquella preponderante en la etapa anterior de la formulación de políticas activas en el país: la de la «capacitación». Mientras que la primera hace mella en las competencias «básicas» o «transversales», es decir, aquellas en relación con las normas más elementales de conexión social (aunque, también por ello, más ancladas en los contextos de socialización de clase de los jóvenes), la segunda se enfoca en la formación de competencias específicas, es decir, en las «calificaciones laborales». Esto se conecta con lo que mencionamos acerca de la promoción de la flexibilidad de los jóvenes para su «mejor» inserción en el mercado de trabajo:

La calificación se circunscribe al puesto, la competencia se centra en la persona que puede llegar a ocupar uno o más puestos. Se puede hablar de un puesto o de un empleo calificado, pero no de un puesto o de un empleo competente, ya que el término implica la intervención de un sujeto. La competencia depende concretamente de la persona y no abstractamente de los requerimientos de un puesto de trabajo. Es el conjunto de herramientas compuestas por conocimientos, habilidades y destrezas con las que cuenta una persona para insertarse y/o desempeñarse en el mundo laboral. Permite el satisfactorio desempeño en situaciones reales de empleo. Reconoce lo que la persona hace realmente y no solamente lo que se le puede pedir como las tareas que atañen a su rol. Las competencias dependen de cada individuo, quien debe movilizarlas, sintetizarlas y ponerlas en acción. (MTESS - Empleabilidad)

De este modo, en relación con el supuesto de la subjetividad como soporte de las intervenciones y de las carencias de los jóvenes vulnerables, la direcciona- 
lidad de las políticas activas se centra en la formación y la orientación, no en un sentido técnico (calificación), sino más bien en el de una formación actitudinal.

Esto aparece muy claramente en los segmentos de los documentos destinados a preparar a los jóvenes para momentos de interacción formal como los de la entrevista laboral. Los consejos y los lineamientos se orientan a regular su propia presentación, borrando todas las marcas estilísticas de clase que pudiesen contribuir a la discriminación social que pesa sobre ellos.

[...] presentación personal: vestimenta neutral, cómoda y con sentido común, adecuada al contexto y al puesto de trabajo, sin ser demasiado llamativa y que pueda mantenerse luego en el ejercicio del puesto. Para ello, será necesario recabar información sobre el estilo habitual de la empresa y del empleo al que se aspira. Considerar (y, por lo tanto, evitar su uso) que la elección de determinados adornos personales, tales como, por ejemplo, maquillajes muy acentuados, aros llamativos, piercings o rastas suelen resultar contraproducentes en determinados lugares y circunstancias y, por lo tanto, disminuir las posibilidades de obtener el empleo. Preparar y revisar la documentación presentada y/o necesaria: documento de identidad, carta de presentación y CV. Es importante releer el CV, fundamentalmente. (MTESS - Manual clubes de empleo)

Con este objetivo, se utilizan eufemismos tales como sentido común o neutro para referirse a códigos estéticos e interaccionales imperantes en los segmentos formales del mercado de trabajo, asociados a las clases medias y dominantes, particularmente exacerbados en el sector de servicios y transmutados en reglas «universales» de las interacciones laborales:

[...] es importante darse a conocer como una persona singular, propositiva, activa, simple, sincera y confiable. Se debe tener en cuenta la actitud mental con la que se concurra a una entrevista: predisposición positiva en todo momento y respecto a todas las preguntas y respuestas. Claridad de objetivos y de la propia identidad laboral y/o profesional. Conciencia de que no existen preguntas acertadas o equivocadas, sino que lo que cuenta es ser conscientes de los propios recursos y potencialidades. Confianza en las capacidades de responder a preguntas y de identificar los intereses propios (MTESS - Manual clubes de empleo)

En este sentido, los materiales apuntan a desarrollar la iniciativa, la confianza, la actitud proactiva y la predisposición mental positiva como valores específicos del ámbito laboral, en detrimento de las lógicas de la «necesidad», la "pasividad» y la «donación", más propias del ámbito de la asistencia. Así, la orientación respecto al modo de comportarse en el momento de las entrevistas hace hincapié en la actitud de «venderse» o, en otras palabras, de ofrecer servicios laborales «negociando» los términos de la relación, antes que «suplicando» por un empleo:

[...] en esta, los postulantes y las postulantes tienen la oportunidad de darse a conocer y de relevar información más concreta acerca de las condiciones del 
puesto de trabajo para el cual se postulan. Es decir, no se trata de una instancia en que es oportuno "suplicar» para obtener el empleo. Adicionalmente, en determinadas circunstancias, podrían darse las condiciones idóneas para negociar ciertos aspectos de los requerimientos planteados por el empleador o la empleadora. (MTESS - Manual clubes de empleo)

El sentido profundamente pedagógico de las políticas activas penetra y configura la mayoría de sus servicios, desde la promoción de la terminalidad educativa formal (que incluye no solo su certificación, sino también el supuesto de una particular experiencia institucional de disciplinamiento) hasta la noción de formación y orientación como aprendizaje. Centrada en las competencias transversales, la política activa de empleo promociona el "aprender a aprender» como noción que articula los elementos anteriormente desarrollados:

Aprender a aprender es una tarea personal, un darse cuenta, un camino de autoconocimiento que se desarrolla en el curso de la vida y que sirve para diversos objetivos, etapas y circunstancias. Aprender a aprender potencia la autonomía requerida por la era tecnológica, donde el flujo constante y múltiple de información, al alcance de cada persona, le requiere desarrollar la capacidad para buscar, seleccionar, priorizar e incorporar los datos, sin depender de que otro se lo enseñe. (MTESS - Manual empleabilidad)

Los relacionadores entrevistados del equipo técnico del PJMYMT aclaran obsesivamente la diferencia entre una "pasantía» y un empleo propiamente dicho ${ }^{10}$. Mientras que el segundo implica una relación de dependencia formal, con una serie de obligaciones y derechos entre las partes, el programa no «inserta laboralmente» a los jóvenes, sino que les abre la posibilidad de tener «experiencias laborales certificadas» o, tal como indica su denominación técnica, «entrenamientos laborales». En base a esta noción centrada en el "aprendizaje», los integrantes del equipo técnico defienden determinadas condiciones y reglas, que van desde el tiempo laboral válido para los jóvenes (que no puede superar la media jornada, aunque la empresa lo deseara), hasta el tipo de tareas que pueden realizar (pasantías que destinan a los beneficiarios a ocupaciones puramente descalificadas son muchas veces dadas de baja por esos motivos). Los jóvenes, en cambio, siempre se toman las pasantías como una oportunidad de "quedar fijo", a partir de lo cual aceptan muchas de las horas extras o el sobretrabajo propuesto por los encargados o los empleadores, con el objetivo de "hacer buena letra» y tener más chances de ser contratado formalmente por la empresa.

La figura de "activación» en este paradigma no se aplica solamente a la activación de tipo económico, sino, fundamentalmente, a la activación actitudinal:

10. En las reformas legales de la década de 1990, la inclusión de diversas modalidades de contratación flexibles sirvió para legitimar, fundamentalmente para los jóvenes «sin experiencia», formas de contratación inestables y precarias que profundizaban las estructuras de desigualdad (Pérez, 2013). 
una formación en valores en relación con el trabajo, que desarrolla competencias - subjetivas - de interacción propias de los empleos formales, aportando a la formación de la autonomía individual de las personas, desaprendiendo códigos culturales construidos en el marco de «familias problemáticas», trayectorias laborales informales e inestables y experiencias de política asistencialista.

\section{Reflexiones finales}

Si bien el paradigma de las políticas activas orientado por la noción de empleabilidad se constituye en un dispositivo de intervención para la totalidad de la fuerza de trabajo, entendemos que la construcción del problema del empleo juvenil tiene un lugar central en la legitimación y puesta en funcionamiento de este cuerpo de documentos de diagnóstico y formación. En este marco, la figura de la juventud vulnerable condensa en sí, a la vez, la carencia —el déficit de competencias-, el momento de transición — homólogo al de la postconvertibilidad- y el peligro —o la necesidad efectiva de intervención para evitar la desintegración y la anomia-. La definición del diagnóstico, en términos de subjetividad individual como soporte de las carencias y las intervenciones, construye un campo de acción para la política de empleo fundamentalmente en términos de formación actitudinal: de educación moral, de disposición para comunicar e interactuar en el «mundo laboral formal» y de adquisición de hábitos positivos para el trabajo. Podríamos referirnos a un campo de acción en términos de una nueva crianza de los jóvenes para el mundo laboral.

En este sentido, podemos hablar, junto a Martín Criado, de "economía moral de las relaciones laborales». Economía, porque implica recursos materiales y juego de inversiones y desinversiones. Moral, porque está ligada a un sistema simbólico que distingue las prácticas legítimas — «buenas»— de las ilegítimas —《malas» (1998: 347).

En los discursos de los integrantes del equipo técnico del PJMYMT, el diagnóstico de la empleabilidad aparece transmutado en términos de un relato social sobre la cultura del trabajo: los jóvenes vulnerables, provenientes de situaciones familiares "problemáticas» y "difíciles», de trayectorias laborales signadas por la «informalidad» y por la «inestabilidad», acostumbrados a la lógica «asistencialista», habrían perdido la cultura del trabajo, constituida no solo por competencias básicas y transversales, sino también, fundamentalmente, por valores. Aquellas actitudes en las que, en tanto recursos, deben ser formados los jóvenes de sectores populares para su transición hacia la vida de adultos como trabajadores dignos: los valores del esfuerzo, de la voluntad, de la proactividad, etc.

En el campo de posibles que se abre en torno a las políticas activas de empleo promovidas por la OIT y el MTESS, así como en las tensiones y en los desplazamientos que van desde la «empleabilidad» hasta la "cultura del trabajo», desde las «competencias y actitudes» hasta los «valores», de la «capacitación» a la «orientación», de la «proactividad» a la «sumisión activa», los jóvenes negocian y definen su valor (económico, en términos salariales, pero también moral y 
simbólico, en términos de dignidad) como sujetos, en un campo económico y moral (el mercado laboral) producido por una red de dispositivos, agenciando y experienciando sus propios problemas (los juveniles) en los términos en los que las políticas se los proponen (Chaves, 2010).

En ese marco de límites y posibilidades, de constreñimientos y aperturas, otra tensión fuerte es la que se establece entre lo que podríamos llamar la «exigencia de autonomía» y la «existencia en la dependencia». Mientras, desde el supuesto de individualidad como modelo de persona en el discurso de la OIT y el MTESS, la "dependencia» cobra sentido negativo y se asocia a aquello que se pretende superar (la infantilidad, la cultura asistencialista), en la cultura popular, al contrario, se es, se existe, precisamente en vínculos con otros, en lazos que ponen en juego derechos y obligaciones mutuas. Entonces, aquí, la dependencia no solo cobra un valor simbólico (moral) positivo, sino que también constituye un recurso material fundamental: un capital social que, en sus formas individual, doméstica o colectiva, es susceptible de ser movilizado en todos los ámbitos de la existencia cotidiana (Gutiérrez, 2004a), incluyendo, claro está, el laboral.

Consideramos que las tensiones que hemos mostrado $-\mathrm{y}$ que, a nuestro entender, derivan de la manera de construir la problemática del empleo juvenil - deben ser puestas en discusión, en la medida en que asumimos que las políticas sociales tienen un rol activo en la construcción de la vida social (Danani, 2004), lo cual contribuye a la conformación de los diferentes modos de reproducción que pueden encontrarse en una sociedad históricamente determinada (Gutiérrez, 2004b; Hintze, 2006).

\section{Referencias bibliográficas}

BASUALDo, Eduardo (2006). «La reestructuración de la economía argentina durante las últimas décadas: De la sustitución de importaciones a la valorización financiera». En: BASUALDO, Eduardo y ARCeo, Enrique (comps.). Neoliberalismo y sectores dominantes: Tendencias globales y experiencias nacionales. Buenos Aires: CLACSO.

Bourdieu, Pierre (1990a). «Espacio social y génesis de las clases». En: Bourdieu, Pierre. Sociología y cultura. México: Grijalbo.

- (1990b). «La “juventud” no es más que una palabra». En: Bourdieu, Pierre. Sociología y cultura. México: Grijalbo.

CAjíAs, Huascar (1999). «Estigma e identidad: Una aproximación a la cuestión juvenil». Última Década, 10, 0.

ChAves, Mariana (2005). «Juventud negada y negativizada: Representaciones y formaciones discursivas vigentes en la Argentina contemporánea». Última Década [en línea], 23, 9-32. $<$ http://dx.doi.org/10.4067/S0718-22362005000200002>.

- (2010). Jóvenes, territorios y complicidades: Una antropología de la juventud urbana. Buenos Aires: Espacio.

Danani, Claudia (2004). «El alfiler en la silla: Sentidos, proyectos y alternativas en el debate de las políticas sociales y la economía social». En: DANANI, Claudia (comp.). Política social y economía social: Debates fundamentales. Buenos Aires: UNGS-OSDE-Altamira. 
Darmon, Isabelle; Frade, Carlos; Demanzière, Didier y Hass, Isabelle (2006). «Formadores y usuarios frente al doble vínculo de la formación para la empleabilidad». Cuadernos de Relaciones Laborales, 24 (2), 37-62.

Dias Duarte, Luiz Fernando (1994). «A outra Saúde: Mental, psicossocial, físicomoral?». En: Alves, Paulo y Minayo, María Cecilia de Souza (orgs.). Saúde e Doença: Um olhar antropológico. Rio de Janeiro: Fiocruz.

- (1995). "Pessoa e Dor no Ocidente (o "holismo metodológico" na Antropologia da Saúde e doença)». Horizontes Antropológicos: Corpo, Doença e Saúde, 4 (9), 13-28.

- (2004). «Las tres configuraciones de la perturbación en Occidente y los nervios de las clases populares». Apuntes de Investigación del CECYP, 9, 75-91.

Foucault, Michel (1985). «¿Qué es la ilustración? (1983)». En: Foucault, Michel. Saber y verdad. Madrid: La Piqueta.

González Bombal, Inés; Kessler, Gabriel y Svampa, Maristella (2010). «Introducción: Las reconfiguraciones del mundo popular». En: GonZÁlez Bombal, Inés; Kessler, Gabriel y Svampa, Maristella (coords.). Reconfiguraciones del mundo popular: El conurbano bonaerense en la posconvertibilidad. Buenos Aires: PrometeoUNGS.

Gutiérrez, Alicia (2004a). Pobre como siempre: Estrategias de reproducción social en la pobreza. Córdoba: Ferreyra.

- (2004b). "La teoría de Bourdieu en la explicación y comprensión del fenómeno de la pobreza urbana». En: Martín Criado, Enrique; Alonso, Luis Enrique y Moreno Pestaña, José Luis (comp.). Pierre Bourdieu: Las herramientas del sociólogo. Madrid: Fundamentos.

- (2011). "Clase, espacio social y estrategias: Una introducción al análisis de la reproducción social en Bourdieu». En: Bourdieu, Pierre. Las estrategias de la reproducción social. Buenos Aires: Siglo XXI.

Hintze, Susana (2006). «Exclusión, derechos y políticas sociales: La promoción de formas asociativas y trabajo autogestivo en la Argentina». Fermentum, 45, 100-137.

Isla, Alejandro y MíGuez, Daniel (2010). Entre la inseguridad y el temor: Instantáneas de la sociedad actual. Buenos Aires: Paidós.

Jacinto, Claudia (2008). «Los dispositivos recientes de empleo juvenil: Institucionalidades, articulaciones con la educación formal y socialización laboral». Revista del Trabajo: Nueva Época, 6, 123-142.

- (2010). «Veinte años de políticas de formación para el empleo de jóvenes vulnerables en América Latina: Persistencias y reformulaciones». En: Jacinto, Claudia (comp.). La construcción social de las trayectorias laborales de jóvenes: Políticas, instituciones, dispositivos y subjetividades. Buenos Aires: Teseo - IDES.

Jacinto, Claudia y MillenaAr, Verónica (2009). «Enfoques de programas para la inclusión laboral de los jóvenes pobres: Lo institucional como soporte subjetivo». Última Década [en línea], 30, 67-92. <http://dx.doi.org/10.4067/S0718-22362009000100004>.

Lenoir, Remi (1993). «Objeto sociológico y problema social». En: Champagne, Patrick et al. Iniciación a la práctica sociológica. México: Siglo XXI.

Lomnitz, Larissa (1978). ¿Cómo sobreviven los marginados? México: Siglo XXI.

- (1979). "Mecanismos de articulación entre el sector informal y el sector formal urbano». En: Tokman, Víctor y Klein, Emilio (comps.). El subempleo en América Latina [en línea]. Buenos Aires: CLACSO-El Cid Editor. <http://dx.doi.org/10.2307/3539837>. 
Martín Criado, Enrique (1998). Producir la juventud: Critica de la sociología de la juventud. Madrid: Itsmo.

- (1999). «El paro juvenil no es el problema, la formación no es la solución». En: CaChón Rodríguez, Lorenzo (coord.). Juventudes, mercados de trabajo y políticas de empleo. Valencia: 7 imig.

- (2005). «La construcción de los problemas juveniles». Nómadas, 23, 86-93.

Merklen, Denis (2005). Pobres ciudadanos: Las clases populares en la era democrática [Argentina 1983-2003]. Buenos Aires: Gorla.

Míguez, Daniel y SEMán, Pablo (2006). «Diversidad y recurrencia en las culturas populares actuales». En: Míguez, Daniel y Semán, Pablo (eds.). Entre santos, cumbias y piquetes: Las culturas populares en la Argentina reciente. Buenos Aires: Biblos.

Neffa, Julio C. (coord.) (2012). Empleo, desempleo y políticas de empleo: De las políticas pasivas a las políticas activas de empleo: análisis comparativo (1989.2011). Buenos Aires: CEIL-PIETTE.

Neffa, Julio C.; Oliveri, María L. y Persia, Juliana (2010). «Transformaciones del mercado de trabajo en la Argentina: 1974-2009». En: Neffa, Julio C.; Panigo, Demián y PÉrez, Pablo (comps.). Transformaciones del empleo en Argentina: Estructura, dinámica e instituciones. Buenos Aires: CICCUS.

Pérez, Pablo (2013). «Inserción laboral juvenil y políticas públicas de empleo en Argentina». Revista Juventude.br. (en prensa).

Plesnicar, Lorena (2013). "'La juventud' en desarrollo en el discurso de los organismos internacionales». Revista Fuente: Nueva Época, 4 (12), 1-12.

Quirós, Julieta (2006). Cruzando la Sarmiento: Una etnografía sobre piqueteros en la trama social del sur del Gran Buenos Aires. Buenos Aires: Antropofagia.

- (2011). El porqué de los que van: Peronistas y piqueteros en el Gran Buenos Aires (una antropología de la politica vivida). Buenos Aires: Antropofagia.

SAlvia, Agustín (2008). "Introducción: La cuestión juvenil bajo sospecha». En: SAlvia, Agustín (comp.). Jóvenes promesas: Trabajo, educación y exclusión social de jóvenes pobres en la Argentina. Buenos Aires: Miño y Dávila.

- (2013). Juventudes, problemas de empleo y riesgos de exclusión social: El actual escenario de crisis mundial en Argentina. Berlín: Friedrich-Ebert-Stiftung.

SEMán, Pablo (2006). «Cosmológica, holista y relacional: Una corriente de la religiosidad popular contemporánea». En: SEMÁn, Pablo. Bajo continuo: Exploraciones descentradas sobre cultura popular y masiva. Buenos Aires: Gorla.

- (2007). "Psicologización y religión en un barrio del Gran Buenos Aires». Debates do NER, 1 (12), 8-44.

VARGAS, Patricia (2005). Bolivianos, paraguayos y argentinos en la obra: Identidades étnico-nacionales entre los trabajadores de la construcción. Buenos Aires: Antropofagia.

Zapata, Laura (2005). La mano que acaricia la pobreza: Etnografía del voluntariado católico. Buenos Aires: Antropofagia.

\section{Fuentes}

Epele, Marisa y SAmpedro, Rosana (2011). Potenciando la empleabilidad de los jóvenes: Conceptos y herramientas para el reconocimiento y desarrollo de ocho competencias laborales. Buenos Aires: Fundación SES-MTESS. (MTESS - Manual empleabilidad)

Fundación SES (2011). Curso para talleristas: Manual del participante. Buenos Aires: MTESS. (MTESS - Manual equipo técnico) 
Koyra, Marcelo y BoAri, Hugo (2011). Enfoques y herramientas para el acompañamiento a jóvenes. Buenos Aires: Fundación SES-MTESS. (MTESS - Manual acompañamiento)

Lepore, Eduardo y Schelser (2005). «Diagnóstico del desempleo juvenil». Trabajo, ocupación y empleo: 2. Trayectorias, negociación colectiva e ingresos. Buenos Aires: MTESS-SSPTYEL. (MTESS - Diagnóstico desempleo juvenil)

Marx Schütz, Jutta (2011). Clubes de empleo para jóvenes. Taller de apoyo a la búsqueda de empleo para jóvenes: Manual de capacitación. Buenos Aires: Programa CEA-OIT. (MTESS - Manual Clubes de empleo)

OIT (2012). Competencias para el empleo. Orientaciones de política: Impulso a la empleabilidad de los jóvenes desfavorecidos. Ginebra: Departamento de Conocimientos Teóricos y Prácticos y Empleabilidad Sector del Empleo. (OIT - Competencias)

- (2012). La crisis del empleo de los jóvenes: jActuemos ya! Conferencia Internacional del Trabajo, 101. a reunión. Ginebra. (OIT - Crisis del empleo juvenil)

- (2013). Tendencias mundiales del empleo juvenil. Una generación en peligro: Resumen ejecutivo. Ginebra. (OIT - Tendencias)

Palmieri, María Paz (2011). El proceso de orientación ocupacional: Características y vicisitudes en el trabajo con jóvenes vulnerables. Buenos Aires: Fundación SES-MTESS. (MTESS - Manual orientación ocupacional)

Programa FORMUJER (2004). Proyecto ocupacional: Una metodología de formación para mejorar la empleabilidad. Buenos Aires: MTESS. (MTESS - Manual proyecto ocupacional)

VIDAL, Sonia y FAmá, Malena (2011). La intermediación laboral con jóvenes: Estrategias, técnicas y aprendizajes. Buenos Aires: Fundación SES-MTESS. (MTESS - Manual intermediación) 